\title{
Conhecimentos básicos de pais de crianças submetidas a tratamento fonoaudiológico quanto à audição e linguagem
}

Basic knowledge of parents of children undergoing speech therapy regarding hearing and language Conocimientos básicos de los padres de niños que reciben terapia del habla con respecto a la audición y el lenguaje

\section{Vanessa Luisa Destro FIDÊNCIO ${ }^{1}$} Isabela de Jesus AZEVEDO ${ }^{2}$

Estefaniely dos Santos MENEZES ${ }^{2}$

Kayce Tuanne Silva CAMPOS $^{2}$

Camila de Castro CORRÊA ${ }^{1}$

${ }^{1}$ Professora Titular do Curso de Fonoaudiologia, Centro Universitário Planalto do Distrito Federal - UNIPLAN, 70297-400, Brasília-DF, Brasil

${ }^{2}$ Curso de graduação em Fonoaudiologia, Centro Universitário Planalto do Distrito Federal - UNIPLAN, 70297-400, Brasília-DF, Brasil

\section{Resumo}

Introdução: Acredita-se que pais de crianças pequenas apresentam poucos conhecimentos a respeito do desenvolvimento normal do comportamento auditivo e de linguagem. Ao mesmo tempo, este conhecimento é indispensável para que, ao perceberem o menor sinal de alteração no comportamento de seu filho quanto a essas habilidades, os pais possam procurar auxílio profissional imediato, de forma a favorecer o diagnóstico e intervenção precoces. Objetivo: Investigar o conhecimento de pais de crianças submetidas a tratamento fonoaudiológico quanto ao desenvolvimento auditivo e de linguagem oral. Método: Foram entrevistados 23 pais de crianças em atendimento fonoaudiológico em uma Clínica Escola. Resultados: $O$ total de $69,5 \%$ demonstrou desconhecimento sobre a época em que a criança deve começar a reagir aos sons. A maioria dos entrevistados acredita que a deficiência auditiva pode acarretar em problemas na fala, voz, comportamento, problemas escolares e emocionais. Grande parte demonstrou ter conhecimento sobre a idade em que a criança deve apresentar as primeiras palavras. No entanto, o mesmo não pôde ser observado quanto à questão da data limite para ocorrência de trocas fonológicas. Do total da amostra, $82,6 \%$ relatou não ter recebido orientações sobre o desenvolvimento da audição e linguagem oral. Conclusão: Conclui-se que os pais de crianças menores de cinco anos apresentam pouco conhecimento sobre os marcos do desenvolvimento da linguagem oral e comportamento auditivo e que há falha quanto à orientação por parte dos profissionais sobre essas questões, reforçando a necessidade da criação de métodos efetivos para esse fim.

Descritores: Inquéritos e Questionários; Pais; Criança; Linguagem Infantil; Audição.

\section{Abstract}

Introduction: It is believed that parents of young children have little knowledge about the normal development of auditory and language behavior. At the same time, this knowledge is indispensable so that, when they perceive the slightest sign of alteration in their child's behavior regarding these abilities, parents can seek immediate professional help in order to favor early diagnosis and intervention. Objective: To investigate the knowledge of parents of children undergoing speech and language therapy regarding the development of hearing and oral language. Methods: We interviewed 23 parents of children in speech therapy at a School Clinic. Results: The total of $69.5 \%$ demonstrated lack of knowledge about the time when the child should begin to react to the sounds. Most of the interviewees (52.2\%) believe that hearing impairment can lead to problems in speech, voice, behavior, school problems and emotional problems. A large proportion (47.9\%) demonstrated that they were aware of the age at which the child should present the first words. However, the same could not be observed regarding the issue of the deadline for occurrence of phonological exchanges. Of the total sample, $82.6 \%$ reported not having received guidance on the development of hearing and oral language. Conclusion: We conclude that the parents of children under five years have little knowledge about the developmental milestones of oral language and auditory behavior and that there is a failure on the guidance from professionals on these issues, reinforcing the need to develop effective methods for this purpose.

Descriptors: Surveys and Questionnaires; Parents; Child; Child Language; Hearing.

\section{Resumen}

Introducción: Se cree que los padres de niños pequeños presentan pocos conocimientos acerca del desarrollo normal del comportamiento auditivo y del lenguaje. Al mismo tiempo, este conocimiento es indispensable para que, al percibir la menor señal de alteración en el comportamiento de su hijo en cuanto a esas habilidades, los padres puedan buscar ayuda profesional inmediata, para favorecer el diagnóstico e intervención temprana. Objetivo: Investigar el conocimiento de padres de niños sometidos a tratamiento fonoaudiológico en cuanto al desarrollo de la audición y el lenguaje. Metodos: Se entrevistó a 23 padres de niños en tratamento fonoaudiologico en una Clinca Escola.Resultados: El total del 69,5\% mostró desconocimiento sobre la época en que el niño debe comenzar a reaccionar a los sonidos. La mayoría de los entrevistados (52,2\%) cree que la deficiencia auditiva puede acarrear problemas en el habla, la voz, el comportamiento, los problemas escolares y emocionales. Gran parte $(47,9 \%)$ demostró tener conocimiento sobre la edad en que el niño debe presentar las primeras palabras. Sin embargo, no se pudo observar la cuestión de la fecha límite para el intercambio de fonemas. Del total de la muestra, el 82,6\% relató no haber recibido orientaciones sobre el desarrollo de la audición y el lenguaje oral. Conclusión: Se concluye que los padres de niños menores de cinco años presentan poco conocimiento sobre los marcos del desarrollo del lenguaje oral y comportamiento auditivo y que hay fallas en la orientación por parte de los profesionales sobre estas cuestiones, reforzando la necesidad de la creación de métodos efectivos para este fin.

Descriptores: Encuestas y Cuestionarios; Padres; Niño; Lenguaje Infantil; Audición.

INTRODUÇÃO

A linguagem desempenha um papel
essencial na organização perceptual,
estruturando as informações para a

aprendizagem e para as relações interpessoais do ser humano. É composta por habilidades receptivas e expressivas, além de ser influenciada por outros fatores, tais como a cognição, audição e as praxias articulatórias ${ }^{1}$.
Os dois primeiros anos de vida são determinantes para a aquisição e desenvolvimento de fala e linguagem, pois esse é o período quando se dará a consolidação das redes neuronais que garantirão o funcionamento adequado dos sistemas sensoriais ${ }^{2,3}$.

$O$ desenvolvimento da linguagem oral ocorre de modo gradativo e sua construção 
inicia-se desde o choro, logo nos primeiros meses de vida, até a elaboração de complexas narrativas (nível pragmático). Esse desenvolvimento está diretamente relacionado a alguns fatores de risco de ordem biológica e ambiental ${ }^{4}$. Um desses fatores de risco é alteração do input sensorial como, por exemplo, a alteração auditiva. A audição é um dos prérequisitos para a aquisição e o desenvolvimento da linguagem. Desta forma, pode-se afirmar que audição e linguagem são funções correlacionadas e interdependentes ${ }^{1-4}$.

O desenvolvimento da audição inicia-se ainda na vida intrauterina, por volta da vigésima semana de gestação ${ }^{5}$. Desde 2010, com a publicação da Lei Federal no 12.303 , é garantida a realização da Triagem Auditiva Neonatal Universal (TANU) em todos os recém-nascidos vivos em território nacional ${ }^{6}$. A realização da TANU é uma estratégia para detectar precocemente alterações auditivas que poderão interferir na qualidade de vida do recémnascido, devendo ocorrer até o primeiro mês de idade ${ }^{5-8}$.

No entanto, além do fato da TANU não atingir $100 \%$ da população de nascidos vivos, existe uma parcela que apresenta a deficiência auditiva, mas passa no teste. Os pais ou cuidadores são as pessoas que passam a maior parte do tempo com a criança, desde o nascimento, sendo os principais responsáveis pela observação do seu comportamento ${ }^{7}$. Desta forma, é necessário o investimento em esclarecimentos a respeito de quais comportamentos devem ser observados e quais são marcos do desenvolvimento normal da criança.

O objetivo do diagnóstico e intervenção precoce no caso da deficiência auditiva é, principalmente, maximizar as competências linguísticas e comunicativas e o desenvolvimento da alfabetização. Sem oportunidades apropriadas para aprender a língua, estas crianças apresentarão atraso quanto ao desenvolvimento da linguagem, cognição e desenvolvimento socioemocional ${ }^{6-8}$.

A maioria dos pais desconhece ou apresenta conhecimentos vagos a respeito dos aspectos relacionados ao desenvolvimento de seus filhos ${ }^{9}$. Ao mesmo tempo, é de extrema importância que os pais conheçam sobre o desenvolvimento normal da linguagem $e$ audição para que, ao perceberem o menor sinal de alteração no comportamento de seu filho quanto a essas habilidades, possam procurar auxílio profissional imediato, de forma a diminuir a possibilidade de um diagnóstico tardio e suas consequências.
Essa importância de que os pais tenham o conhecimento sobre o desenvolvimento típico auditivo e de linguagem também se fundamenta em pesquisas anteriores que demonstraram a necessidade de ampliar os conhecimentos sobre esta temática expressada por profissionais, como pediatras, enfermeiros e cirurgiões-dentistas para aprimorar para suas condutas como o encaminhamento precoce ao fonoaudiólogo ${ }^{10-11}$.

Diante do exposto, o objetivo deste estudo foi investigar o conhecimento de pais de crianças submetidas ao tratamento fonoaudiológico quanto ao desenvolvimento da audição e linguagem.

MATERIAL E MÉTODO

- Aspectos éticos

Este estudo teve início após aprovação do Comitê de Ética em Pesquisas da União Educacional do Planalto Central UNICEPLAC/DF sob processo $n=2.982 .364$. Os participantes assinaram 0 Termo de Consentimento Livre e Esclarecido (TCLE), atestando sua participação no estudo.

- Casuística

Inicialmente, foram abordados 30 acompanhantes de crianças atendidas na Clínica Escola de Fonoaudiologia do Centro Universitário Planalto do Distrito Federal (UNIPLAN). Para inclusão na pesquisa, os participantes deveriam ser maiores de 18 anos e ter filho(s) com idade entre 0 e 4 anos e 11 meses que estivesse $(\mathrm{m})$ em atendimento de terapia fonoaudiológica. Destes, um participante foi excluído por ser avô e não pai da criança e outros 6 foram excluídos por serem pais de crianças que já haviam completado 5 anos de idade, resultando então na casuística final de 23 participantes. Na Tabela 1, estão apresentadas as características sociodemográficas dos pais entrevistados.

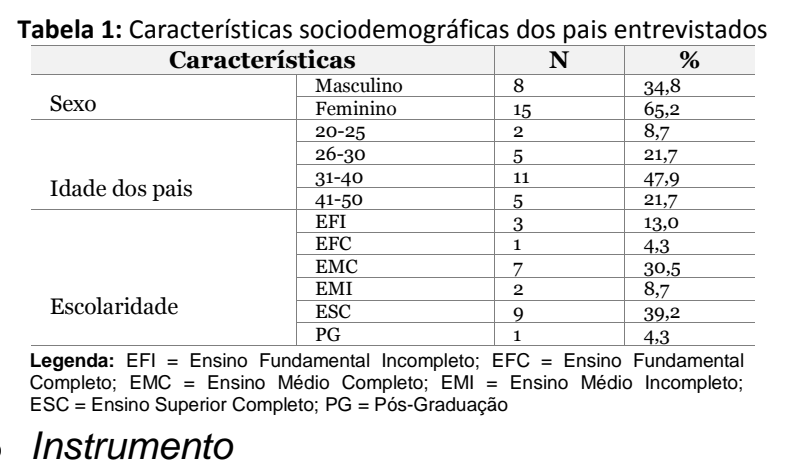

Os participantes responderam a um questionário estruturado (Apêndice A), composto por 20 questões dissertativas $e$ objetivas (múltipla escolha), adaptado de Wolff e Goulart ${ }^{11}$. Além dos tópicos contidos no questionário base, foram elaboradas questões 
extras que abordaram aspectos do nível de informação e sobre a queixa dos pais, relacionados à linguagem e audição (Quadro 1). Ressalta-se que as alterações realizadas e descritas no Quadro 1 fizeram-se necessárias para que fosse possível atingir o objetivo proposto no presente estudo.

Quadro 1. Adaptações realizadas pelas autoras do presente estudo do questionário Wolff e Goulart ${ }^{11}$.

\begin{tabular}{|c|c|c|}
\hline & $\begin{array}{l}\text { Componente do } \\
\text { questionário }\end{array}$ & $\begin{array}{l}\text { Adaptações realizadas pelas } \\
\text { autoras do presente estudo }\end{array}$ \\
\hline & Identificação & $\begin{array}{l}\text { O item "escolaridade" foi } \\
\text { transformado de questão escrita } \\
\text { para múltipla escolha; } \\
\text { O item "área de trabalho" foi } \\
\text { excluído. }\end{array}$ \\
\hline $\begin{array}{l}\text { Questões } \\
\text { baseadas no } \\
\text { questionário de } \\
\text { Wolff e Goulart }^{11}\end{array}$ & $\begin{array}{l}\text { Nível de } \\
\text { Informação }\end{array}$ & $\begin{array}{l}\text { O item "Nível de Informação" } \\
\text { passou a ser o item "III" do } \\
\text { questionário; } \\
\text { As questões } 8,9,10,11 \text { e } 14 \text { foram } \\
\text { excluídas; } \\
\text { A questão "6" passou a ser a } \\
\text { questão "10"; } \\
\text { A questão "7" passou a ser a } \\
\text { questão "11"; } \\
\text { A questão "12" passou a ser a } \\
\text { questão "17"; } \\
\text { A questão "13" passou a ser a } \\
\text { questão "18" e o termo "problema } \\
\text { auditivo" foi substituído pelo } \\
\text { termo "deficiência auditiva" }\end{array}$ \\
\hline $\begin{array}{l}\text { Questões } \\
\text { elaboradas pelas } \\
\text { autoras r e } \\
\text { acrescentadas ao } \\
\text { questionário }\end{array}$ & $\begin{array}{l}\text { Queixa } \\
\text { Nível de } \\
\text { Informação }\end{array}$ & $\begin{array}{l}\text { Acrescentou-se o Componente II - } \\
\text { "Queixa" ao questionário, com } 4 \\
\text { questões }(6,7,8 \text { e 9); } \\
\text { No Componente III - "Nível de } \\
\text { Informação", acrescentou-se as } \\
\text { questões } 12,13,14,15,16,19 \text { e } 20 \text {. }\end{array}$ \\
\hline
\end{tabular}

RESULTADOS

Os resultados apresentados a seguir descrevem uma análise qualitativa dos dados encontrados neste estudo. Observou-se que a maioria dos pais procurou atendimento por conta própria e a queixa principal foi "atraso na fala" (Tabela 2).

Tabela 2. Dados referentes à queixa principal, encaminhamento e indicação da Clínica Escola.

\begin{tabular}{l|c|c|c}
\hline \multirow{4}{*}{ Queixa principal } & & $\mathbf{N}$ & $\mathbf{\%}$ \\
\cline { 2 - 4 } & Atraso na fala & $\mathbf{1 1}$ & 47,9 \\
\cline { 2 - 4 } & Trocas fonológicas & 4 & 17,4 \\
\cline { 2 - 4 } & Gagueira & $\mathbf{1}$ & 4,3 \\
\cline { 2 - 4 } & Outros comprometimentos & 2 & 8,7 \\
\cline { 2 - 4 } Encaminhamento & Não relatou & 5 & 21,7 \\
\hline & Médico Pediatra & 9 & 39,2 \\
\cline { 2 - 4 } & Escola & $\mathbf{1}$ & 4,3 \\
\cline { 2 - 4 } & Fonoaudiólogo & 2 & 8,7 \\
\cline { 2 - 4 } & Procurou por conta própria & 11 & 47,9 \\
\hline \multirow{4}{*}{$\begin{array}{l}\text { Indicação da } \\
\text { Clínica Escola }\end{array}$} & Amigos e familiares & 9 & 39,2 \\
\cline { 2 - 4 } & Escola & 2 & 8,7 \\
\cline { 2 - 4 } & Outras clínica/hospitais & 3 & 13,0 \\
\cline { 2 - 4 } & Internet & 2 & 8,7 \\
\cline { 2 - 4 } & Funcionários do Centro & 3 & 13,0 \\
\cline { 2 - 4 } & Universitário & & \\
\cline { 2 - 4 } & Estudantes do Centro & 4 & 17,4 \\
\hline
\end{tabular}

$\mathrm{Na}$ Tabela 3 estão apresentados os dados referentes à percepção dos pais quanto ao início do desenvolvimento da audição e a idade em que julgam ser possível iniciar a avaliação audiológica.

Quanto à ocorrência de avaliação audiológica, de acordo com o relato dos pais, todas as crianças já haviam sido submetidas a algum exame. Do total da amostra, 47,9\% relataram que seus filhos foram submetidos somente à TANU, não tendo realizado nenhuma avaliação auxiológica no decorrer dos primeiros 5 anos de vida.
Outras 8,7\% realizaram, além da TANU, avaliação audiológica na própria Clínica Escola. Um total de $43,5 \%$ dos participantes afirmou que seus filhos não foram submetidos à TANU, mas já realizaram avaliação audiológica, sendo que destas, somente em um caso a mãe procurou espontaneamente esta avaliação. As outras crianças foram submetidas à avaliação audiológica somente após serem encaminhadas por algum profissional.

Quanto à importância da audição para o desenvolvimento, dos 23 participantes, somente um $(4,3 \%)$ respondeu acreditar que a audição não é essencial para o desenvolvimento da linguagem, sendo necessária para "ouvir música". Todos os outros participantes responderam que acreditam que o sentido é essencial para o desenvolvimento da criança como um todo, incluindo desenvolvimento da linguagem e comunicação.

Somente $17,4 \% \quad(n=19) \quad$ relataram já terem recebido orientações quanto ao desenvolvimento auditivo e de linguagem oral. Os demais $82,6 \%$ dos entrevistados relataram não terem recebido orientações sobre essas questões, mesmo após início do tratamento fonoaudiológico. Destes $(n=19)$, dois entrevistados $(10,5 \%)$ relataram que sempre comparam o desempenho do filho que está em tratamento fonoaudiológico com os irmãos ou outras crianças da família e outros dois $(10,5 \%)$ relataram já terem recebido orientações quanto ao modo de estimular a linguagem da criança no ambiente domiciliar, mas não quanto aos marcos do desenvolvimento da linguagem ou quais habilidades devem observar na criança em cada fase do desenvolvimento.

Tabela 3. Percepção dos pais quanto ao desenvolvimento da audição e idade mínima para a realização de avaliação audiológica

\begin{tabular}{c|c|c|c}
\hline \multicolumn{2}{c|}{ Percepção dos pais (Nível de Informação) } & $\mathbf{N}$ & $\mathbf{\%}$ \\
\hline & ao nascimento & 7 & 30,5 \\
Idade em que a criança deve & $1-3$ & 1 & 4,3 \\
começar a reagir aos sons (meses) & $3-6$ & 8 & 34,8 \\
& $6-9$ & 2 & 8,7 \\
& $9-12$ & 1 & 4,3 \\
& Acima de 12 & 4 & 17,4 \\
\hline & ao nascimento & 6 & 26,1 \\
& antes de 1 ano & 8 & 34,8 \\
Idade mínima em que é possível & $1-2$ anos & 1 & 4,3 \\
realizar avaliação audiológica (anos) & $2-3$ anos & 2 & 8,7 \\
& $3-4$ anos & 3 & 13,0 \\
& não sabe & 3 & 13,0 \\
\hline
\end{tabular}

DISCUSSÃO

mais Segundo pesquisas ${ }^{12-14}$, as demandas fonoaudiológica em crianças estão relacionadas aos problemas na fala, corroborando com os resultados do presente estudo. Embora alguns autores preconizem as trocas fonológicas ${ }^{15}$, observou-se que esta foi uma queixa relatada apenas por $17,4 \%(n=4)$ dos pais.

Conforme o exposto, $47,9 \%(n=11)$ dos 
entrevistados procuraram atendimento fonoaudiológico para seu(a) filho(a) por conta própria. Em segundo lugar, vieram aqueles encaminhados pelo médico pediatra, que constituíram $37,2 \%(n=9)$ da amostra. Observase, portanto, que grande parte dos entrevistados percebeu, sem auxílio profissional, que poderia haver alguma alteração no desenvolvimento de linguagem de seu(a) filho(a). Durante as entrevistas observaram-se relatos corriqueiros de pais que procuraram atendimento espontaneamente julgando ser normal a alteração apresentada pela criança, mas alegando que outras pessoas sugeriram procurar pelo fonoaudiólogo. Além disso, também notou-se que os pais alegam como motivo da procura pelo tratamento fonoaudiológico o fato de observarem que o outro filho ou filho de um amigo apresentou o desenvolvimento diferente.

A maioria dos pais entrevistados, 47,9\% $(n=11)$, demonstrou ter conhecimento sobre a idade em que a criança deve apresentar as primeiras palavras, visto que sabe-se que, no geral, entre nove e dezoito meses de vida as crianças com desenvolvimento típico apresentam o início das primeiras palavras ${ }^{16}$.

Para cada faixa etária são esperados determinados processos fonológicos, de acordo com o desenvolvimento auditivo da criança e de sua exposição à estrutura da língua materna ${ }^{17}$. Até os sete anos espera-se o último processo fonológico na fala da criança ${ }^{17}$. Observou-se que apenas $4,3 \%(n=1)$ respondeu que a idade limite para a ocorrência de trocas na fala é de sete anos, corroborando com o estudo ${ }^{17}$. Os demais entrevistados demonstraram desconhecimento do assunto e, aparentemente, do comportamento do desenvolvimento fonológico da criança, pois uns não souberam responder $(4,3 \%)$ à pergunta, outros alegaram não ser normal em nenhuma idade $(8,7 \%)$ e a outra parte $(30,4 \%)$ afirmou ser o limite abaixo dos 6 anos de idade.

No tocante ao conhecimento dos pais sobre a idade em que as crianças devem começar a reagir aos sons, 30,5\% $\quad(n=7)$ consentiram que ao nascer a criança já apresente essa habilidade; em segundo plano, $69,5 \%(n=16)$ demonstraram desconhecimento de quando a criança deve começar a reagir aos sons. Em 2001, um estudo comprovou que o feto é capaz de responder a estímulos sonoros, demonstrando que antes do nascimento a criança já pode reagir a eles ${ }^{18}$.

Foi possível observar que $47,9 \%$ dos entrevistados $(n=11)$ responderam que seu filho foi submetido à TANU, contudo, somente $26,1 \%$ $(n=6)$ responderam que é possível avaliar a audição da criança ao nascer. Nota-se, portanto, que é possível que esses pais não tenham recebido orientações adequadas sobre a avaliação a qual seu(a) filho(a) estava sendo submetido. Em uma investigação anterior realizada com 132 famílias em uma maternidade, os autores constataram que $15 \%$ não tiveram conhecimento à respeito da existência da TANU em nenhum momento, $24 \%$ souberam da triagem auditiva na alta hospitalar, $22 \%$ durante a internação, $12 \%$ depois da alta hospitalar, $4 \%$ antes da internação, e as demais famílias (23\%) não souberam informar ${ }^{19}$.

No Art. 5ำ do Código de Ética da Fonoaudiologia $^{20}$ consta que uma das competências do fonoaudiólogo é o trabalho de orientação. Além das orientações quanto à avaliação que está sendo realizada, as explicações para pais de crianças submetidas à TANU com e sem indicadores de risco, segundo as Diretrizes de Atenção da Triagem Auditiva Neonatal, ${ }^{21}$ devem ser sobre o comportamento auditivo e de linguagem da criança, ou seja, sobre os marcos de desenvolvimento. É de extrema importância que a família compreenda como ocorre o desenvolvimento normal da audição e da linguagem, a fim de entender o que deve ser esperado em cada fase e saber identificar qualquer alteração. Há na literatura a indicação de uma cartilha a ser entregue após a realização da TANU, onde é dito, de forma resumida, o procedimento que a criança está passando e o resultado. As possibilidades do resultado são explicadas de forma simplória. Ao lado, os autores trazem orientações sobre os marcos do desenvolvimento da audição e sua implicação no desenvolvimento da linguagem ${ }^{22}$.

No presente estudo, observou-se que dez crianças $(43,5 \%)$ não foram submetidas à TANU. Considerando a idade das crianças cujos pais foram entrevistados ( 0 a 5 anos), esse dado evidencia que a detecção precoce da deficiência auditiva ainda não é uma realidade em todos os lugares, mesmo após a publicação da Lei Federal $\mathrm{n}^{\circ}{ }^{12.303^{6}}$, que garante a realização do teste.

Observou-se que $82,6 \% \quad(n=19)$ dos participantes relataram não terem recebido orientações sobre 0 desenvolvimento de audição e linguagem, mesmo após início do tratamento fonoaudiológico. Além do já exposto acima quanto às competências do fonoaudiólogo no âmbito da orientação ${ }^{19}$, é evidenciado em literatura que os indivíduos precisam ter conhecimento e serem esclarecidos quanto ao tratamento, para que possam contribuir para o sucesso terapêutico ${ }^{23}$. 
Não basta somente orientar os pais quanto às estratégias que podem ser realizadas em casa para a estimulação da criança. A orientação deve também ter o caráter de informá-los detalhadamente quanto à alteração apresentada por seu filho e sobre quais competências esperar da criança em cada fase do seu desenvolvimento.

O significado de saúde como ausência de doença tem ganhado outras definições. Atualmente pode ser definido como um estado completo de bem-estar físico, mental e social, resultando na capacidade de enfrentar adoecimentos, buscar ajuda e entender o que está acontecendo ${ }^{24}$. A saúde é determinada por fatores sociais, econômicos, culturais, ambientais, étnico-raciais, psicológicos, fisiológicos e políticos ${ }^{24-26}$. Artigos confirmam o conhecimento de que as condições sociais das famílias determinam o estado de saúde das crianças $^{25,27}$, e as condições sociais são atribuídas ao nível de escolaridade e de instrução de sua família. Para a produção da saúde das crianças, a escolaridade dos pais é tida como um fator de muita relevância ${ }^{28}$, levando-os a apresentar níveis melhores de saúde, a pensar o que é melhor e do que seu filho precisa ${ }^{29-30}$.

Como decorrência de outras pesquisas, observam-se que pais de classe social e nível escolar mais elevados ${ }^{31}$ ou mais baixos ${ }^{32}$ têm influência na informação e produção de saúde da criança, contudo, para a presente pesquisa, foi um dado um tanto quanto irrelevante quando voltado o olhar para a questão socioeconômica dos pais como determinante da saúde da criança, já que estes pais estão classificados em diferentes níveis sociais, incluindo escolaridade e socioeconômico.

Dos quatro $(17,4 \%)$ participantes que afirmaram terem recebido orientações sobre 0 desenvolvimento normal da linguagem, um relatou que essa orientação foi realizada por uma profissional da enfermagem. Esse relato aparece em outro estudo ${ }^{19}$, no qual constatouse que as enfermeiras foram as profissionais mais citadas pelas famílias entrevistadas em maternidades, quando questionadas sobre quem as orientou quanto à TANU. As autoras justificaram este achado com o fato de que essas profissionais possuem maior contato com as mães no período neonatal e reforçam a necessidade de que haja maior capacitação para estas profissionais com relação à saúde auditiva. Diante do resultado obtido no presente estudo, observa-se a necessidade de que sejam também capacitadas com relação aos marcos do desenvolvimento de linguagem da criança, porém sem que interfiram nas competências que cabem ao profissional fonoaudiólogo.

Em 2007, um estudo realizado com mães de bebês pré-termos, verificou que as orientações não estavam sendo fornecidas de maneira sistemática e padronizada ${ }^{33}$ e que as participantes relataram que a criação de um instrumento de orientação seria um importante suporte para prover e minimizar os medos e suprir as expectativas quanto ao desenvolvimento da criança.

Adquirir a linguagem é ser bombardeado constantemente pelos sons da língua, é aprender que os objetos e pessoas têm nomes que são constituídos de sons específicos que se seguem em sequência. A criança que escuta, inconscientemente adquire a estrutura da gramática de sua língua e pode combinar e recombinar os elementos linguísticos definidamente35. Quanto ao conhecimento da implicação da audição ou da falta dela no desenvolvimento da criança, $52,2 \%(n=12)$ dos participantes atestaram ciência de que a deficiência auditiva pode ocasionar problemas na fala, voz, comportamento, problemas escolares e emocionais. Uma entrevistada $(4,3 \%)$ alegou que a audição não é importante para a fala, em vista que sua filha escuta tudo o que as pessoas dizem, mas ela não verbaliza. A colocação da participante evidenciou a falta de orientação quanto ao desenvolvimento auditivo e de linguagem.

Outro ponto importante observado neste estudo é que, quando há orientação aos pais,

Outro ponto importante observado neste estudo é que, quando há orientação aos pais, essa discorre sobre o desenvolvimento de linguagem, de modo separado dos marcos do desenvolvimento auditivo.

CONCLUSÃO

Conclui-se que os pais de crianças menores de 5 anos submetidas a atendimento fonoaudiológico apresentam déficit nos conhecimentos básicos sobre o desenvolvimento da linguagem oral e comportamento auditivo e que se faz necessária a elaboração, por parte dos profissionais fonoaudiólogos, de estratégias de orientação a esse público.

\section{REFERÊNCIAS}

1. Costa MPR. Análise comparativa da linguagem oral de crianças ouvintes e surdas usuárias de implante coclear. Rev CEFAC. 2009;11(4): 662-72.

2. Nascimento GB, Kessler TM, Souza APR, Costa I, Moraes AB. Risk indicators for hearing loss and language acquisition and their 
relationship with socioeconomic, demographic and obstetric variables in preterm and term babies. Codas. 2020;10;32(1):e20180278.

3. Friederici AD. The neural basis of language development and its impairment. Neuron. 2006; 52(6):941-52.

4. Panes ACS, Corrêa CC, Maximino LP. Checklist para identificação de crianças de risco para alterações de linguagem oral: nova proposta. Distúrb Comum. 2018;30(2):278-87.

5. Pujol R, Lavigne-Rebillard M. Development of neurosensory structures in the human cochlea. Acta Otolaryngol. 1992;112(2):259-64.

6. Brasil. Presidência da República Lei 12.303 de 2 de agosto de 2010. Dispõe sobre a obrigatoriedade de realização do exame denominado Emissões Otoacústicas Evocadas. [Internet]. Brasília, DF; 2010. [acesso em 2018 nov. 10]. Disponível em: http://www2.camara.leg.br/legin/fed/lei/2010/lei12303-2-agosto-2010-607594publicacaooriginal-128606-pl.html

7. Onoda RM, Azevedo MF, Santos AMN. Neonatal hearing screening: failures, hearing loss and risk indicators. BJORL. 2011;77(6): 775-83.

8. American Academy of Pediatrics, Joint Committee on Infant Hearing. Year 2007 position statement: Principles and guidelines for early hearing detection and intervention programs. Pediatrics. 2007;120(4):898-921.

9. Wolff GS, Goulart BNG. Percepção dos pais sobre os distúrbios fonoaudiológicos na infância. Rev bras crescimento desenvolv. 2013;23(2):177-83.

10. Maximino LP, Ferreira MV, Oliveira DT, Lamônica DAC, Feniman MR, Spinardi ACP, Lopes-Herrera SA. Conhecimentos, atitudes e práticas dos médicos pediatras quanto ao desenvolvimento da comunicação oral. Rev CEFAC. 2009;11(Supl2):267-74.

11. Pizolato RA, Fonseca LMM, Bastos RS, Fernandes AY, Lefévre $F$, Maximino LP. Vigilância do desenvolvimento da linguagem da criança: conhecimentos e práticas de profissionais da atenção básica à saúde. Rev CEFAC. 2016;18(5):1109-112.

12. César AM, Maksud SS. Caracterização da demanda de fonoaudiologia no serviço público municipal de ribeirão das neves - MG. Rev CEFAC. 2007;9(1):133-38.

13. Diniz RD, Bordin R. Demanda em Fonoaudiologia em um serviço público municipal da região Sul do Brasil. Rev Soc Bras Fonoaudiol. 2011;16(2):126-31.

14. Mandrá PP, Diniz MV. Caracterização do perfil diagnóstico e fluxo de um ambulatório de Fonoaudiologia hospitalar na área de linguagem infantil. Rev Soc Bras Fonoaudiol. 2011;16(2):121-25.

15. Barros PML, Oliveira PN. Perfil dos pacientes atendidos no setor de Fonoaudiologia de um serviço público de recife - PE. Rev. CEFAC. 2010;12(1):128-33.

16. Van Riper C, Emerick L. Correção da linguagem. 8.ed. Porto Alegre: Artes Médicas; 1997.

17. Prates LPCS, Martins VO. Distúrbios da fala e da linguagem na infância. Rev méd Minas Gerais. 2011 21(4-S1):54-60.

18. Moon C. The role of early auditory development in attachment and communication. Clin Perionatol. 2011;38(4):657-69.

19. Alvarenga KF, Gadret JM, Araújo ES, Bevilacqua MC. Triagem auditiva neonatal: motivos da evasão das famílias no processo de detecção precoce. Rev Soc Bras Fonoaudiol. 2012;17(3):241-47.

20. Código de Ética da Fonoaudiologia. Brasília: $11^{\circ}$ Colegiado do CFFa, 2016.

21. Ministério da Saúde. Diretrizes de Atenção da Triagem Auditiva Neonatal. Brasília: MS, 2012.

22. Nishino LK, Guilherme AS. Manual de audiologia pediátrica. São Paulo: Manole; 2015.

23. Souza SCMES. Esclarecimento de pais e/ou responsáveis quanto ao Tratamento fonoaudiológico realizado em crianças: enfoque bioético [monografia]. Florianópolis: Universidade Federal de Santa Catarina-UFSC; 2015.

24. Ministério da Saúde. Saúde e doença: dois fenômenos da vida. Rio de Janeiro: MS, 2005.

25. Buss PM, Filho AP. A Saúde e seus Determinantes sociais. Rev Saúde Coletiva. 2007;17(1):77-93.

26. Souza DO, Silva SEV, Silva NO. Determinantes Sociais da Saúde: reflexões a partir das raízes da "questão social. Saúde Soc, 2013;22(1): 44-56.

27. Ceballos AGC, Cardoso C. Determinantes sociais de alterações fonoaudiológicas. Rev Soc Bras Fonoaudiol. 2009;14(3):441-45.

28. Alves D, Belluzzo W. Infant mortality and child health in Brazil. Econ Hum Biol. 2004;2(3): 391-410.

29. Santos AMA, Tejada CAO, Ewerling F. Os determinantes socioeconômicos do estado de saúde das crianças do Brasil rural. RESR. 2012;50(3):473-92.

30. Ribas JRRC, Moura MLS, Bornstein MH. Cognições maternas acerca da maternidade e do desenvolvimento humano: uma contribuição ao estudo da psicologia parental. Rev Bras Cresc Desenvolv Hum. 2007;17(1):104-13.

31. Cardoso RM, Pedromonico MRM, Silva EMK, Puccini RF. Conhecimento de mães e auxiliares de desenvolvimento infantil referente ao desenvolvimento da linguagem de crianças de zero a 4 anos. Rev Bras Cresc Desenv Hum. 2003;13(2):83-91.

32. Peixoto MVS, Siqueira CGA, Silva AF, Pedruzzi CM, Santos AA. Caracterização da população 
assistida por um serviço de Fonoaudiologia em uma Unidade de Saúde. Distúrb Comum. 2010; 22(2):107-15.

33. Martinez CMS, Joaquim RHVT, Oliveira EB, Santos IC. Suporte informacional como elemento para orientação de pais de pré-termo: um guia para o serviço de acompanhamento do desenvolvimento no primeiro ano de vida. Rev Bras Fisioter. 2007;11(1):73-81.

34. Conselho Federal de Fonoaudiologia. Áreas de competência do fonoaudiólogo no Brasil. Documento Oficial, 2.ed. Mar. 2007.

35. Jakubovicz R. Avaliação, diagnóstico e tratamento em

Fonoaudiologia: psicomotricidade, deficiência de audição, atraso de linguagem simples e gagueira infantil. 2.ed. Rio de Janeiro; Revinter: 2002.

\section{APÊNDICE}

- Instrumento de coleta de dados

I - Identificação

1- Sexo:

(1) masculino

(2) feminino

2- Idade:

2- Escolaridade:

(1) analfabeto

(2) ensino fundamental completo

(3) ensino fundamental incompleto

(4) ensino médico completo

(5) ensino médio incompleto

(6) ensino superior completo

(7) ensino superior incompleto

(8) pós-graduação

4- Quantos filhos?

5 - Idade dos filhos?

II - Queixa

6 - Sexo do filho atendido na clínica escola: (1) masculino

7 - Idade do filho atendido na clínica escola:

(1) espontaneamente

(2) encaminhado. Neste caso, por quem?

9 - Como soube da clínica escola?

III - Nível de informação

10 - Até que idade considera normal que a criança troque as letras na fala? 11 - Com que idade aproximada a criança deve começar a falar?

12 - Com que idade aproximada a crianç deve começar a reagir aossons?

13 - Apresenta casos de surdez na família? (1) sim

14 - A criança atendida na clínica escola já realizou alguma avaliação audiológica?

(1) $\operatorname{sim}$

(2) não

(3) somente triagem auditiva neonatal ("teste da orelhinha)

Caso tenha respondido sim, procurou a avaliação:

(1) espontaneamente

(2) encaminhada

15 - Algum outro membro da família já realizou avaliação audiológica alguma vez?

(1) sim. Quem?

(2) não

16- A partir de qual idade acredita que é possível avaliar a audição da criança?

17 - Acredita que a audição é importante para:

(1) aprender a falar

(2) comunicar-se

(3) desenvolvimento da criança como um todo

(4) ouvir música

(5) outro. Qual?

18 - Acredita que uma criança com deficiência auditiva pode apresentar:

(1) problemas na fala

(2) problemas escolare

(3) problemas na voz

(4) problemas escolares

(5) problemas emocionais

(6) problemas comportamentais

(7) nenhuma das opções

(8) outro. Qual?

19 - Você já recebeu alguma orientação sobre o desenvolvimento da audição da criança?

(1) sim. Onde?

(2) não

20 - Você já recebeu alguma orientação sobre o desenvolvimento da linguagem da criança?

(1) sim. Onde?

(2) não

\section{CONFLITO DE INTERESSES}

Os autores declaram não haver conflitos de interesse AUTOR PARA CORRESPONDÊNCIA

Vanessa Luisa Destro Fidêncio

Av. Pau Brasil, s/n, lote 2, Águas Claras, 70297-400, Brasília-DF, Brasil

E-mail: vanessa.destrof@gmail.com 